\title{
Transcendental Singularities for a Meromorphic Function with Logarithmic Derivative of Finite Lower Order
}

\author{
J. K. Langley ${ }^{1}$
}

Received: 1 March 2018 / Revised: 23 July 2018 / Accepted: 25 July 2018 / Published online: 10 September 2018 (c) The Author(s) 2018

\begin{abstract}
It is shown that two key results on transcendental singularities for meromorphic functions of finite lower order have refinements which hold under the weaker hypothesis that the logarithmic derivative has finite lower order.
\end{abstract}

Keywords Meromorphic function - Direct and indirect transcendental singularities . Logarithmic derivative

Mathematics Subject Classification 30D35

\section{Introduction and Results}

Suppose that $f$ is a transcendental meromorphic function on $\mathbb{C}$ such that, as $z$ tends to infinity along a path $\gamma$ in the plane, $f(z)$ tends to some $\alpha \in \mathbb{C}$. Then, for each $t>0$, an unbounded subpath of $\gamma$ lies in a component $C(t)$ of the set $\{z \in \mathbb{C}$ : $|f(z)-\alpha|<t\}$. Here, $C(t) \subseteq C(s)$ if $0<t<s$, and the intersection $\bigcap_{t>0} C(t)$ is empty [2]. The path $\gamma$ then determines a transcendental singularity of the inverse function $f^{-1}$ over the asymptotic value $\alpha$ and each $C(t)$ is called a neighbourhood of the singularity $[2,18]$. Two transcendental singularities over $\alpha$ are distinct if they have disjoint neighbourhoods for some $t>0$. Following [2,18], a transcendental singularity of $f^{-1}$ over $\alpha \in \mathbb{C}$ is said to be direct if $C(t)$, for some $t>0$, contains finitely many points $z$ with $f(z)=\alpha$, in which case there exists $t_{1}>0$ such that $C(t)$ contains no $\alpha$-points of $f$ for $0<t<t_{1}$. A direct singularity over $\alpha \in \mathbb{C}$ is logarithmic if there exists $t>0$ such that $\log t /(f(z)-\alpha)$ maps $C(t)$ conformally onto the right half plane. If, on the other hand, $C(t)$ contains infinitely many $\alpha$-points

\section{Communicated by Walter Bergweiler.}

$凶 \quad$ J. K. Langley

james.langley@nottingham.ac.uk

1 School of Mathematical Sciences, University of Nottingham, Nottingham NG7 2RD, UK 
of $f$, for every $t>0$, then the singularity is called indirect: a well-known example is given by $f(z)=z^{-1} \sin z$, with $\alpha=0$ and $\gamma$ the positive real axis $\mathbb{R}^{+}$. Transcendental singularities of $f^{-1}$ over $\infty$ and their corresponding neighbourhoods may be defined and classified using $1 / f$, and the asymptotic and critical values of $f$ together comprise the singular values of $f^{-1}$.

If $f$ has finite (lower) order of growth, as defined in terms of the Nevanlinna characteristic function $T(r, f)[8,18]$, then the number of direct singularities is controlled by the celebrated Denjoy-Carleman-Ahlfors theorem $[9,18]$.

Theorem 1.1 (Denjoy-Carleman-Ahlfors theorem) Let $f$ be a transcendental meromorphic function in the plane of finite lower order $\mu$. Then the number of direct transcendental singularities of $f^{-1}$ is at most $\max \{1,2 \mu\}$.

A key consequence of Theorem 1.1 is that a transcendental entire function of finite lower order $\mu$ has at most $2 \mu$ finite asymptotic values [9]. A result of Bergweiler and Eremenko [2] shows that the critical values of a meromorphic function of finite (lower) order have a decisive influence on indirect transcendental singularities.

Theorem 1.2 [2] Let $f$ be a transcendental meromorphic function in the plane of finite lower order.

(a) If $f^{-1}$ has an indirect transcendental singularity over $\alpha \in \widehat{\mathbb{C}}=\mathbb{C} \cup\{\infty\}$, then each neighbourhood of the singularity contains infinitely many zeros of $f^{\prime}$ which are not $\alpha$-points of $f$; in particular, $\alpha$ is a limit point of critical values of $f$.

(b) If $f$ has finitely many critical values, then $f^{-1}$ has finitely many transcendental singularities, and all transcendental singularities are logarithmic.

Theorem 1.2 was proved in [2] for $f$ of finite order and was extended to finite lower order, using essentially the same method, by Hinchliffe [11]. Part (b) follows from part (a) combined with Theorem 1.1 and a well-known classification theorem from [18, p. 287], which shows in particular that any transcendental singularity of the inverse function over an isolated singular value is logarithmic. Theorem 1.2 was employed in [2] to prove a long-standing conjecture of Hayman [7] concerning zeros of $f f^{\prime}-1$, and has found many subsequent applications, including zeros of derivatives [12]. The reader is referred to $[3,19]$ for further striking results on singularities of the inverse, both restricted to entire functions but independent of the order of growth.

The starting question of the present paper concerns the extent to which Theorems 1.1 and 1.2 hold under the weaker hypothesis that $f^{(k)} / f$ has finite lower order for some $k \in \mathbb{N}=\{1,2, \ldots\}$. The obvious example $f(z)=\exp (\exp (z))$ shows that $f^{\prime} / f$ can have finite order despite $f$ having infinite lower order; here, $f^{-1}$ has infinitely many direct (indeed logarithmic) singularities over 0 and $\infty$, and one over 1. Furthermore, if $k \in \mathbb{N}$ and $A_{k}$ is a transcendental entire function, then the lemma of the logarithmic derivative [8] shows that every non-trivial solution of

$$
w^{(k)}-A_{k}(z) w=0
$$

has infinite lower order, even if $A_{k}$ has finite order. Clearly, each of $\exp (\exp (z))$ and $\exp \left(z^{-1} \sin z\right)$ satisfies an equation of form (1.1) with coefficient of finite order. Note 
further that if $f$ is a transcendental meromorphic function in the plane and $f^{\prime} / f$ has finite lower order, then it is easy to prove by induction that so has $A_{k}=f^{(k)} / f$ for every $k \geq 1$, using the formula $A_{k+1}=A_{k} A_{1}+A_{k}^{\prime}$, whereas the example

$$
f(z)=e^{-z / 2} \sin \left(e^{z}\right), \quad \frac{f^{\prime}(z)}{f(z)}=-\frac{1}{2}+e^{z} \cot \left(e^{z}\right), \quad \frac{f^{\prime \prime}(z)}{f(z)}=\frac{1}{4}-e^{2 z}
$$

shows that $f^{\prime \prime} / f$ can have finite order despite $f^{\prime} / f$ having infinite lower order.

Theorem 1.3 Let $f$ be a transcendental meromorphic function in the plane such that $f^{-1}$ has $n \geq 1$ distinct direct transcendental singularities over finite non-zero values. Let $k \in \mathbb{N}$ and let $\mu$ be the lower order of $A_{k}=f^{(k)} / f$. Then the following statements hold.

(i) There exists a set $F_{0} \subseteq[1, \infty)$ of finite logarithmic measure such that

$$
\lim _{r \rightarrow+\infty, r \notin F_{0}} \frac{\log \left(\min \left\{\left|A_{k}(z)\right|:|z|=r\right\}\right)}{\log r}=-\infty .
$$

(ii) If $n \geq 2$, then $n \leq 2 \mu$.

(iii) If $n=1$ and there exist $\kappa>0$ and a path $\gamma$ tending to infinity in the complement of the neighbourhood $C(\kappa)$ of the singularity, then $\mu \geq 1 / 2$.

Theorem 1.3 will be deduced from a version of the Wiman-Valiron theory for meromorphic functions with direct tracts developed in [4], and part (ii) is sharp, by Example 1 in Sect. 2. Furthermore, if $g$ is a transcendental entire function of lower order less than $1 / 2$, then the inverse function of $f=1-1 / g$ has a direct singularity over 1 ; in this case, $A_{k}$ obviously has lower order less than $1 / 2$, but the $\cos \pi \lambda$ theorem $[9, \mathrm{Ch}$. 6] implies that every neighbourhood of the singularity contains circles $|z|=r$ with $r$ arbitrarily large, so that a path $\gamma$ as in (iii) cannot exist.

Theorem 1.4 Let $f$ be a transcendental meromorphic function in the plane such that $f^{(k)} / f$ has finite lower order for some $k \in \mathbb{N}$. Assume that $f^{-1}$ has an indirect transcendental singularity over $\alpha \in \widehat{\mathbb{C}}$. Then each neighbourhood of the singularity contains infinitely many zeros of $f^{\prime} f^{(k)}$, which are not $\alpha$-points of $f$.

Theorem 1.4 will be proved using a modification of methods from $[2,11]$.

Corollary 1.1 Let $f$ be a transcendental meromorphic function in the plane, with finitely many critical values, such that $f^{\prime} / f$ has finite lower order. Then $f^{-1}$ has finitely many transcendental singularities over finite non-zero values, and $f$ has finitely many asymptotic values. Moreover, all transcendental singularities of $f^{-1}$ are logarithmic.

Corollary 1.1 follows from Theorems 1.3 and 1.4, coupled with [18, p. 287].

Corollary 1.2 Let $f$ be a transcendental meromorphic function in the plane such that $f^{\prime \prime} / f$ has lower order $\mu<\infty$ and $f^{\prime} / f$ and $f^{\prime \prime} / f^{\prime}$ have finitely many zeros. Then $f^{\prime \prime} / f^{\prime}$ is a rational function and $f$ has finite order and finitely many poles. 
To prove Corollary 1.2, observe that all but finitely many zeros of $f^{\prime} f^{\prime \prime}$ are zeros of $f$. Thus, $f^{-1}$ has no indirect singularities, by Theorem 1.4 , and hence $f$ has finitely many asymptotic values, in view of Theorem 1.3. Since $f$ evidently has finitely many critical values, the result follows via [12, Theorem 2]. The condition $\mu<\infty$ holds if $f^{\prime} / f$ has finite lower order, and is not redundant, because of an example in [12].

The last result of this paper is related to the following theorem from [14].

Theorem 1.5 [14] Let $M$ be a positive integer and let $f$ be a transcendental meromorphic function in the plane with transcendental Schwarzian derivative

$$
S_{f}(z)=\frac{f^{\prime \prime \prime}(z)}{f^{\prime}(z)}-\frac{3}{2}\left(\frac{f^{\prime \prime}(z)}{f^{\prime}(z)}\right)^{2},
$$

such that: (i) $f$ has finitely many critical values and all multiple points of $f$ have multiplicity at most $M$; (ii) the inverse function of $f$ has finitely many transcendental singularities.

Then the following three conclusions hold: (a) $f$ has infinitely many multiple points; (b) the inverse function of $S_{f}$ does not have a direct transcendental singularity over $\infty$; (c) the value $\infty$ is not Borel exceptional for $S_{f}$.

Conclusion (a) is a result of Elfving [6] and Nevanlinna [17,18], but was proved in [14] by a completely different method. The following example shows that under the hypotheses of Theorem 1.5 the inverse of the Schwarzian can have a direct transcendental singularity over a finite value: write

$$
g(z)=\sinh z, \quad S_{g}(z)=1-\frac{3 \tanh ^{2} z}{2},
$$

so that $S_{g}^{-1}$ has two logarithmic singularities over $-1 / 2$. However, assumptions (i) and (ii) of Theorem 1.5 imply that $f$ belongs to the Speiser class $\mathcal{S}$ [1,2] consisting of all meromorphic functions in the plane for which the inverse function has finitely many singular values. For $f \in \mathcal{S}$, the following result excludes direct singularities of the inverse of $S_{f}$ over 0 .

Theorem 1.6 Let $f$ be a transcendental meromorphic function in the plane belonging to the Speiser class $\mathcal{S}$, with transcendental $S c h w a r z i a n$ derivative $S_{f}$. Then the inverse function of $S_{f}$ does not have a direct transcendental singularity over 0 .

The example $f(z)=\tan ^{2} \sqrt{z}$ from [5] shows that for $f \in \mathcal{S}$ it is possible for 0 to be an asymptotic value of $S_{f}$. Here direct computation shows that $f^{\prime \prime}(z) / f^{\prime}(z)$ tends to 0 as $z \rightarrow \infty$ in the left half plane, and so does $S_{f}(z)$.

The author thanks the referees for their helpful comments.

\section{Examples Illustrating Theorems 1.3 and 1.4}

Example 1 A function extremal for Theorem 1.3(ii), but not for Theorem 1.1, is given by 


$$
f(0)=1, \quad \frac{f^{\prime}(z)}{f(z)}=\frac{\pi z}{\sin \pi z} .
$$

Here $f$ is meromorphic in the plane, having at each non-zero integer $n$ a zero or pole of multiplicity $|n|$, depending on the sign and parity of $n$. Hence $N(r, f)$ and $N(r, 1 / f)$ have order 2. Because

$$
\begin{aligned}
0<\alpha= & \int_{0}^{+\infty} \frac{\pi y}{\sinh \pi y} \mathrm{~d} y=\int_{0}^{+\infty} \frac{\pi y}{\pi y+(\pi y)^{3} / 6+\cdots} \mathrm{d} y \\
& <\int_{0}^{1} 1 \mathrm{~d} y+\int_{1}^{\infty} \frac{6}{\pi^{2} y^{2}} \mathrm{~d} y<\pi
\end{aligned}
$$

and $f^{\prime} / f$ is even, $f$ has distinct asymptotic values $e^{ \pm i \alpha}$, approached as $z$ tends to infinity along the imaginary axis. As $f^{\prime} / f$ has finite order and $f$ has no finite nonzero critical values, both of these singularities of $f^{-1}$ are direct by Theorem 1.4.

Example 2 Define $g$ by

$$
g(0)=1, \quad \frac{g^{\prime}(z)}{g(z)}=A_{1}(z)=\frac{1}{\pi \cos \sqrt{z}} .
$$

The zeros of $\cos \sqrt{z}$ occur where $\sqrt{z}=b_{n}=(2 n+1) \pi / 2$, with $n \in \mathbb{Z}$, and the residue of $A_{1}$ at $b_{n}^{2}$ is $\pm(2 n+1)$. Thus $g$ is meromorphic in $\mathbb{C}$, with zeros and poles in $\mathbb{R}^{+}$ and no finite non-zero critical values. Integration along the negative real axis shows that $g$ has a non-zero real asymptotic value $\alpha$, and $g^{-1}$ has a logarithmic singularity over $\alpha$ by Corollary 1.1. This gives $\delta>0$ and a simply connected component $C$ of $\{z \in \mathbb{C}:|g(z)-\alpha|<\delta\}$ with $(-\infty, R) \subseteq C$ for some $R<0$. Moreover, $C$ is symmetric with respect to $\mathbb{R}$, since $g$ is real meromorphic, so that $C \cap \mathbb{R}^{+}$is bounded, and $g$ is extremal for Theorem 1.3(iii).

Example 3 Let $F(z)=\exp (-z / 2-(1 / 4) \sin 2 z) \cos z$, so that $F^{\prime \prime} / F$ is entire of finite order. Then $F(z)$ tends to 0 along $\mathbb{R}^{+}$and this singularity of $F^{-1}$ is evidently indirect.

Example 4 Define entire functions $A_{1}$ and $v$ by

$$
v(0)=1, \quad \frac{v^{\prime}(z)}{v(z)}=A_{1}(z)=\frac{1-\cos z}{z^{2}}=\frac{1}{2}+\cdots .
$$

Then there exists $\alpha \in \mathbb{R}^{+}$such that $v(x) \rightarrow \exp ( \pm \alpha)$ as $x \rightarrow \pm \infty$ on $\mathbb{R}$ and, since $A_{1}$ does not satisfy (1.2), Theorem 1.3 implies that $v^{-1}$ has no direct singularities over finite non-zero values. Because all critical points of $v$ are real, all but finitely many of them belong to neighbourhoods of the indirect singularities over $\exp ( \pm \alpha)$, and so $v^{-1}$ has no other indirect singularities, by Theorem 1.4. Thus applying [18, p. 287] again, in conjunction with Iversen's theorem, shows that $v^{-1}$ has logarithmic singularities over the omitted values 0 and $\infty$. 
Example 5 Let $h(z)=\exp (\sin z-z)$, so that $A_{1}=h^{\prime} / h$ is entire of finite order but does not satisfy (1.2). Since $h(z)$ tends to 0 along $\mathbb{R}^{+}$, and to $\infty$ on the negative real axis, with $h^{\prime}(2 \pi n)=0$ for all $n \in \mathbb{Z}$, these singularities of $h^{-1}$ are direct but not logarithmic.

\section{Preliminaries}

The following well-known estimate may be found in Theorem 8.9 of [9].

Lemma 3.1 [9] Let $D_{1}, \ldots, D_{n}$ be $n \geq 2$ pairwise disjoint plane domains. If $u_{1}, \ldots, u_{n}$ are non-constant subharmonic functions on $\mathbb{C}$ such that $u_{j}$ vanishes outside $D_{j}$, then

$$
\liminf _{r \rightarrow \infty} \frac{h(r)}{r^{n / 2}}>0, \quad h(r)=\max _{1 \leq j \leq n} B\left(r, u_{j}\right), \quad B\left(r, u_{j}\right)=\sup \left\{u_{j}(z):|z|=r\right\} .
$$

For $a \in \mathbb{C}$ and $R>0$ denote by $D(a, R)$ the open disc of centre $a$ and radius $R$, and by $S(a, R)$ its boundary circle.

Lemma 3.2 To each $k \in \mathbb{N}$ corresponds $d_{k} \in(0, \infty)$ with the the following property. Suppose that $0<R<\infty$ and $w=h(z)$ maps the domain $U \subseteq \mathbb{C}$ conformally onto $D(a, R)$, with inverse function $F: D(a, R) \rightarrow U$. Then there exists an analytic function $V_{k}: D(a, R) \rightarrow \mathbb{C}$ with

$$
h^{(k)}(z) F^{\prime}(w)^{k}=V_{k}(w), \quad\left|V_{k}(w)\right| \leq \frac{d_{k}}{(R-|w-a|)^{k-1}} \quad \text { as } \quad|w-a| \rightarrow R-.
$$

Proof Assume that $a=0$ and initially that $R=1$. It is clear that (3.2) holds for $k=1$, with $V_{1}(w)=1$. If (3.2) holds for $k$, then it follows that

$h^{(k+1)}(z) F^{\prime}(w)^{k+1}=V_{k}^{\prime}(w)-k h^{(k)}(z) F^{\prime}(w)^{k-1} F^{\prime \prime}(w)=V_{k}^{\prime}(w)-k V_{k}(w) \frac{F^{\prime \prime}(w)}{F^{\prime}(w)}$.

Since $F^{\prime \prime}(w) / F^{\prime}(w)=O(1-|w|)^{-1}$ as $|w| \rightarrow 1-$ by [10, p. 5, (1.6)], applying Cauchy's estimate for derivatives to $V_{k}$ proves the lemma by induction when $R=1$. In the general case write $w=h(z)=R H(z)=R v$ and $z=F(w)=G(v)$ so that, as $|w| \rightarrow R-$,

$$
\left|h^{(k)}(z) F^{\prime}(w)^{k}\right|=R^{1-k}\left|H^{(k)}(z) G^{\prime}(v)^{k}\right| \leq \frac{d_{k} R^{1-k}}{(1-|v|)^{k-1}}=\frac{d_{k}}{(R-|w|)^{k-1}} .
$$


Lemma 3.3 Let $M \in \mathbb{N}$ and $s>2^{24}$ and let $E_{1}, \ldots, E_{N}$ be $N \geq 24 M$ pairwise disjoint domains in $\mathbb{C}$, and for $t>0$ let $\phi_{j}(t)$ be the angular measure of $S(0, t) \cap E_{j}$. Then at least $N-12 M$ of the $E_{j}$ satisfy

$$
\int_{\left[4 s^{1 / 2}, s / 4\right]} \frac{\pi \mathrm{d} t}{t \phi_{j}(t)}>M \log s \text { and } \int_{\left[4 s, s^{2} / 4\right]} \frac{\pi \mathrm{d} t}{t \phi_{j}(t)}>M \log s .
$$

Proof This is a standard application as in [9, Ch. 8] or [2] of the Cauchy-Schwarz inequality, which gives

$$
\frac{L^{2}}{t} \leq \frac{1}{t}\left(\sum_{j=1}^{L} \phi_{j}(t)\right)\left(\sum_{j=1}^{L} \frac{1}{\phi_{j}(t)}\right) \leq 2 \sum_{j=1}^{L} \frac{\pi}{t \phi_{j}(t)}
$$

for $M \leq L \leq N$ and $t>0$. If $s>2^{24}$ and either inequality of (3.3) fails for $L \geq 6 M$ of the $E_{j}$, without loss of generality for $j=1, \ldots, L$, then integrating (3.4) yields a contradiction via

$$
2 L M \log s<6 L M \log \frac{\sqrt{s}}{16} \leq L^{2} \log \frac{\sqrt{s}}{16} \leq 2 L M \log s .
$$

Lemma 3.4 [1] Let h be a transcendental meromorphic function in the plane belonging to the Speiser class $\mathcal{S}$. Then there exist positive constants $C, R$ and $M$ such that

$$
\left|\frac{z h^{\prime}(z)}{h(z)}\right| \geq C \log ^{+}\left|\frac{h(z)}{M}\right| \text { for }|z| \geq R \text {. }
$$

\section{Proof of Theorem 1.3}

Let $f$ be a transcendental meromorphic function in the plane such that $f^{-1}$ has $n \geq 1$ direct singularities over (not necessarily distinct) finite non-zero values $a_{1}, \ldots, a_{n}$. Let $k \in \mathbb{N}$; then $A_{k}=f^{(k)} / f$ does not vanish identically. There exist a small positive $\delta$ and non-empty components $D_{j}$ of $\left\{z \in \mathbb{C}:\left|f(z)-a_{j}\right|<\delta\right\}$, for $j=1, \ldots n$, such that $f(z) \neq a_{j}$ on $D_{j}$, so that $D_{j}$ immediately qualifies as a direct tract for $g_{j}=\delta /\left(f-a_{j}\right)$ in the sense of [4, Section 2]. Here $\delta$ may be chosen so small that if $n \geq 2$ then these $D_{j}$ are pairwise disjoint. For each $j$, define a non-constant subharmonic function $u_{j}$ on $\mathbb{C}$ by

$$
u_{j}(z)=\log \left|g_{j}(z)\right|=\log \left|\frac{\delta}{f(z)-a_{j}}\right| \quad\left(z \in D_{j}\right), \quad u_{j}(z)=0 \quad\left(z \notin D_{j}\right) .
$$

Then [4, Theorem 2.1] implies that, with $B\left(r, u_{j}\right)$ as in (3.1),

$$
\lim _{r \rightarrow+\infty} \frac{B\left(r, u_{j}\right)}{\log r}=+\infty, \quad \lim _{r \rightarrow+\infty} a\left(r, u_{j}\right)=+\infty, \quad a\left(r, u_{j}\right)=r B^{\prime}\left(r, u_{j}\right) .
$$


Lemma 4.1 There exists a set $F_{0} \subseteq[1, \infty)$, of finite logarithmic measure, such that for each $s \in[1, \infty) \backslash F_{0}$ and each $j$ there exists $z_{j}$ with

$$
\left|z_{j}\right|=s, \quad A_{k}\left(z_{j}\right)=\frac{f^{(k)}\left(z_{j}\right)}{f\left(z_{j}\right)}=O\left(\exp \left(-B\left(s, u_{j}\right) / 2\right)\right) .
$$

Proof Fix $\tau$ with $1 / 2<\tau<1$ and apply the version of Wiman-Valiron theory developed in [4] for meromorphic functions with direct tracts. By [4, Theorem 2.2 and Lemma 6.10], there exists a set $F_{0} \subseteq[1, \infty)$ of finite logarithmic measure such that every $s \in[1, \infty) \backslash F_{0}$ has the following two properties: first, $a\left(s, u_{j}\right)$ is large, by (4.1), but satisfies

$$
a\left(s, u_{j}\right) \leq B\left(s, u_{j}\right)^{2}
$$

second, for each $j$ there exists $z_{j}$ with $\left|z_{j}\right|=s$ and $u\left(z_{j}\right)=B\left(s, u_{j}\right)$ such that

$$
\frac{f(z)-a_{j}}{f\left(z_{j}\right)-a_{j}} \sim\left(\frac{z}{z_{j}}\right)^{-a\left(s, u_{j}\right)} \text { for }\left|z-z_{j}\right|<\frac{s}{a\left(s, u_{j}\right)^{\tau}} .
$$

A standard application of Cauchy's estimate for derivatives in (4.4) now gives

$$
\begin{aligned}
\left(\frac{f^{\prime}}{f-a_{j}}\right)^{(p)}(z)= & O\left(\frac{a\left(s, u_{j}\right)}{s}\right)^{p+1} \text { for } p=0, \ldots, k-1 \\
& \text { and }\left|z-z_{j}\right|<\frac{s}{2 a\left(s, u_{j}\right)^{\tau}} .
\end{aligned}
$$

It follows via [8, Lemma 3.5] that

$$
\frac{f^{(k)}\left(z_{j}\right)}{f\left(z_{j}\right)}=\frac{f^{(k)}\left(z_{j}\right)}{f\left(z_{j}\right)-a_{j}} \cdot \frac{f\left(z_{j}\right)-a_{j}}{f\left(z_{j}\right)}=O\left(\frac{a\left(s, u_{j}\right)^{k} \exp \left(-B\left(s, u_{j}\right)\right)}{s^{k}}\right),
$$

which, by (4.3), yields (4.2) for large enough $s \notin F_{0}$.

Combining (4.1) with (4.2) for $j=1$ leads to (1.2). To prove the remaining assertions it may be assumed that $A_{k}$ has finite lower order $\mu$. Choose a positive sequence $\left(r_{m}\right)$ tending to infinity such that

$$
T\left(8 r_{m}, A_{k}\right)<r_{m}^{\mu+o(1)}
$$

Let $m$ be large and let $w_{1}, \ldots, w_{q_{m}}$ be the zeros and poles of $A_{k}$ in $r_{m} / 4 \leq|z| \leq 4 r_{m}$, repeated according to multiplicity: then (4.5) and standard estimates yield

$$
q_{m} \leq n\left(4 r_{m}, A_{k}\right)+n\left(4 r_{m}, 1 / A_{k}\right) \leq \frac{2}{\log 2} T\left(8 r_{m}, A_{k}\right)+O(1) \leq r_{m}^{\mu+o(1)}
$$


Let $U_{m}$ be the union of the discs $D\left(w_{j}, r_{m}^{-\mu}\right)$. Since the sum of the radii of the discs of $U_{m}$ is $o\left(r_{m}\right)$ by (4.6), there exists a set $E_{m} \subseteq\left[r_{m} / 2,2 r_{m}\right]$, of linear measure at least $r_{m}$, and so logarithmic measure $l_{m} \geq 1 / 2$, such that for $r \in E_{m}$ the circle $|z|=r$ does not meet $U_{m}$. A standard application of the Poisson-Jensen formula [8] on the disc $|\zeta| \leq 4 r_{m}$ then yields

$$
|\log | A_{k}(z)|| \leq r_{m}^{\mu+o(1)} \text { for }|z| \in E_{m} .
$$

Since $m$ is large and $l_{m} \geq 1 / 2$, there exists $s_{m} \in E_{m} \backslash F_{0}$.

Suppose now that $n=1$ and there exist $\kappa>0$ and a path $\gamma$ tending to infinity in the complement of the neighbourhood $C(\kappa)$ of the singularity, or that $n \geq 2$. Then (3.1) holds, by [9, Theorem 6.4] when $n=1$, and by Lemma 3.1 when $n \geq 2$. Combining (3.1) and (4.2), with $s=s_{m} \geq r_{m} / 2$, yields points $z_{j}$ with $\left|z_{j}\right|=s_{m}$ and, for at least one $j$,

$$
A_{k}\left(z_{j}\right)=O\left(\exp \left(-B\left(s_{m}, u_{j}\right) / 2\right)\right)=O\left(\exp \left(-s_{m}^{n / 2-o(1)}\right)\right)
$$

On combination with (4.7), this forces $2 \mu \geq n$.

\section{Indirect Singularities}

Proposition 5.1 Let $f$ be a transcendental meromorphic function in the plane such that $f^{(k)} / f$ has finite lower order $\mu$ for some $k \in \mathbb{N}$. Assume that $f^{-1}$ has an indirect transcendental singularity over $\alpha \in \mathbb{C} \backslash\{0\}$. Then for each $\delta>0$, the neighbourhood $C(\delta)$ of the singularity contains infinitely many zeros of $f^{\prime} f^{(k)}$.

The proof of Proposition 5.1 will take up the whole of this section. The method is adapted from those in [2,11], but some complications arise, in particular when $k \geq 2$. Assume throughout that $f$ and $\alpha$ are as in the hypotheses, but $C(\varepsilon)$, for some small $\varepsilon>0$, contains finitely many zeros of $f^{\prime} f^{(k)}$. It may be assumed that $\alpha=1$, and that $C(\varepsilon)$ contains no zeros of $f^{\prime} f^{(k)}$. Choose positive integers $N_{1}, N_{2}, \ldots, N_{9}$ with $5 \mu+12<N_{1}$ and $N_{j+1} / N_{j}$ large for each $j$.

Lemma 5.1 For each $j \in\left\{1, \ldots, N_{9}\right\}$ there exist $z_{j} \in C(\varepsilon)$ and $a_{j} \in \mathbb{C}$ with $0<r_{j}=$ $\left|1-a_{j}\right|<\varepsilon / 2$, as well as a simply connected domain $D_{j} \subseteq C(\varepsilon)$, with the following properties. The $a_{j}$ are pairwise distinct and the $D_{j}$ pairwise disjoint. Furthermore, the function $f$ maps $D_{j}$ univalently onto $D\left(1, r_{j}\right)$, with $z_{j} \in D_{j}$ and $f\left(z_{j}\right)=1$. Moreover, $0 \notin D_{j}$ but $D_{j}$ contains a path $\sigma_{j}$ tending to infinity, which is mapped by $f$ onto the half-open line segment $\left[1, a_{j}\right)$, with $f(z) \rightarrow a_{j}$ as $z \rightarrow \infty$ on $\sigma_{j}$.

This is proved exactly as in [2]. If $0<T_{j}<\varepsilon / 2$ and $z_{j} \in C\left(T_{j}\right)$ is such that $f\left(z_{j}\right)=1$, let $r_{j}$ be the supremum of $t>0$ such that the branch of $f^{-1}$ mapping 1 to $z_{j}$ admits unrestricted analytic continuation in $D(1, t)$. Then $r_{j}<T_{j}$ because $f$ is not univalent on $C\left(T_{j}\right)$, and there is a singularity $a_{j}$ of $f^{-1}$ with $\left|1-a_{j}\right|=r_{j}$; moreover, $a_{j}$ must be an asymptotic value of $f$. The $z_{j}$ and $T_{j}$ are then chosen inductively: for the details see [2] (or [13, Lemma 10.3]). 
Lemma 5.2 Let the $z_{j}, a_{j}, \sigma_{j}$ and $D_{j}$ be as in Lemma 5.1. For $t>0$, let $t \theta_{j}(t)$ be the length of the longest open arc of $S(0, t)$ which lies in $D_{j}$. Then $f$ satisfies, as $z$ tends to infinity on $\sigma_{j}$,

$$
\log \frac{r_{j}}{\left|f(z)-a_{j}\right|} \geq \int_{\left|z_{j}\right|}^{|z|} \frac{\mathrm{d} t}{4 t \theta_{j}(t)}
$$

Proof Let $z=H(w)$ be the branch of $f^{-1}$ mapping $D\left(1, r_{j}\right)$ onto $D_{j}$. For $z \in \sigma_{j}$, the distance from $z$ to $\partial D_{j}$ is at most $|z| \theta_{j}(|z|)$. Thus Koebe's quarter theorem [10, Ch. 1] implies that

$$
\left|\left(w-a_{j}\right) H^{\prime}(w)\right| \leq 4|z| \theta_{j}(|z|) \text { for } z=H(w), w \in\left[1, a_{j}\right)
$$

Hence, for large $z \in \sigma_{j}$ and $w=f(z)$, writing $u=H(v)$ for $v \in[1, w]$ gives (5.1) via

$$
\begin{aligned}
\log \frac{r_{j}}{\left|f(z)-a_{j}\right|} & =\int_{[1, w]} \frac{|\mathrm{d} v|}{\left|a_{j}-v\right|}=\int_{H([1, w])} \frac{|\mathrm{d} u|}{\left|\left(a_{j}-v\right) H^{\prime}(v)\right|} \\
& \geq \int_{H([1, w])} \frac{|\mathrm{d} u|}{4|u| \theta_{j}(|u|)}
\end{aligned}
$$

Since $N_{1}>5 \mu$, there exists a positive sequence $\left(s_{n}\right)$ tending to infinity such that

$$
T\left(s_{n}^{5}, f^{(k)} / f\right)+T\left(s_{n}^{5}, f / f^{(k)}\right) \leq s_{n}^{N_{1}} .
$$

Set

$$
G(z)=z^{N} \frac{f^{(k)}(z)}{f(z)}, \quad N=N_{5}
$$

Applying [15, Lemma 4.1] to $1 / G$ (with $\psi(t)=t$ in the notation of [15]) gives a small positive $\eta$ such that $G$ has no critical values $w$ with $|w|=\eta$ and such that the length $L(r, \eta, G)$ of the level curves $|G(z)|=\eta$ lying in $D(0, r)$ satisfies

$$
L\left(s_{n}^{4}, \eta, G\right)=O\left(s_{n}^{6} T\left(e^{8} s_{n}^{4}, G\right)^{1 / 2}\right)=O\left(s_{n}^{6+N_{1} / 2}\right) \leq s_{n}^{N_{1}} \quad \text { as } n \rightarrow \infty,
$$

using (5.2) and the fact that $N_{1}>12$. Assume henceforth that $n$ is large.

Lemma 5.3 At least $N_{8}$ of the domain $D_{j}$ and paths $\sigma_{j}$, without loss of generality $D_{1}, \ldots, D_{N_{8}}$ and $\sigma_{1}, \ldots, \sigma_{N_{8}}$, are such that

$$
\left|f(z)-a_{j}\right| \leq s_{n}^{-N_{7}} \text { for } z \in \sigma_{j} \text { with }|z| \geq s_{n} / 4 \text {. }
$$


Proof By Lemma 3.3, it may be assumed that, for $j=1, \ldots, N_{8}$,

$$
\int_{\left[4 s_{n}^{1 / 2}, s_{n} / 4\right]} \frac{\pi \mathrm{d} t}{t \theta_{j}(t)}>N_{8} \log s_{n},
$$

which, on combination with Lemma 5.2, leads to (5.5).

Lemma 5.4 Let $w_{1}, \ldots, w_{q_{n}}$ be the zeros and poles of $G$ in $s_{n}^{1 / 4} \leq|z| \leq s_{n}^{4}$, repeated according to multiplicity. Then

$$
q_{n} \leq n\left(s_{n}^{4}, 1 / G\right)+n\left(s_{n}^{4}, G\right)=o\left(s_{n}^{N_{1}}\right)
$$

and there exist $t_{n}, T_{n}$ satisfying

$$
s_{n}^{1 / 2}-1 \leq t_{n} \leq s_{n}^{1 / 2}, \quad s_{n}^{2} \leq T_{n} \leq s_{n}^{2}+1,
$$

such that

$$
\max \left\{|\log | G(z)||: z \in S\left(0, t_{n}\right) \cup S\left(0, T_{n}\right)\right\} \leq s_{n}^{N_{1}+1} .
$$

Proof (5.6) follows from (5.2). Let $U_{n}$ be the union of the discs $D\left(w_{q}, s_{n}^{-N_{1}-1}\right)$ : these discs have sum of radii at most $s_{n}^{-1}$ and so since $n$ is large there exist $t_{n}, T_{n}$ satisfying (5.7) such that the circles $S\left(0, t_{n}\right), S\left(0, T_{n}\right)$ do not meet $U_{n}$. Hence the Poisson-Jensen formula gives (5.8).

Lemma 5.5 Define sets $E, K_{n}$ and $L_{n}$ by $E=\{z \in \mathbb{C}:|G(z)|<\eta\}$ and

$$
K_{n}=\left\{z \in \mathbb{C}: t_{n}<|z|<T_{n}\right\}, \quad L_{n}=\left\{z \in \mathbb{C}: s_{n} / 4<|z|<4 s_{n}\right\} .
$$

Then the number of components $E_{q}$ of $E \cap K_{n}$ which meet $L_{n}$ is at most $s_{n}^{N_{1}}$.

Proof If the closure $F_{q}$ of $E_{q}$ lies in $K_{n}$, then $E_{q}$ must contain a zero of $G$, whereas if $F_{q} \nsubseteq K_{n}$ then $\partial E_{q} \cap K_{n}$ has arc length at least $s_{n} / 8$. Thus the lemma follows from (5.4) and (5.6).

Lemma 5.6 Let $u$ lie on $\sigma_{j}$ with $s_{n} / 4 \leq|u| \leq 4 s_{n}$. Then, with $d_{k}$ as in Lemma 3.2, there exists $v$ on $\sigma_{j}$ such that:

$$
\begin{aligned}
& |u| \leq|v| \leq|u|+s_{n}^{-N_{3}} ; \quad\left|f(v)-a_{j}\right| \leq\left|f(u)-a_{j}\right| \\
& \left|f^{(k)}(v)\right| \leq k^{k} d_{k} s_{n}^{k N_{3}}\left|f(u)-a_{j}\right| .
\end{aligned}
$$

Proof Starting at $u$, follow $\sigma_{j}$ in the direction in which $\left|f(z)-a_{j}\right|$ decreases. Then $\sigma_{j}$ describes an arc $\gamma$ joining the circles $S(0,|u|)$ and $S\left(0,|u|+s_{n}^{-N_{3}}\right)$, such that the first two inequalities of (5.9) hold for all $v \in \gamma$. Since $f$ maps $D_{j}$ univalently onto $D\left(1, r_{j}\right)$, the inverse function $H$ of $f$ maps a proper sub-segment $I$ of the half-open 
line segment $J=\left[f(u), a_{j}\right)$ onto $\gamma$. Assume that the last inequality of (5.9) fails for all $v \in \gamma$. Then Lemma 3.2 yields, on $I$,

$$
\left|H^{\prime}(w)\right| \leq k^{-1} s_{n}^{-N_{3}}\left|f(u)-a_{j}\right|^{-1 / k}\left(r_{j}-|w-1|\right)^{1 / k-1} .
$$

Since $1, f(u)$ and $a_{j}$ are collinear, a contradiction arises via

$$
\begin{aligned}
s_{n}^{-N_{3}} \leq\left|\int_{I} H^{\prime}(w) \mathrm{d} w\right| & \leq \int_{I} k^{-1} s_{n}^{-N_{3}}\left|f(u)-a_{j}\right|^{-1 / k}\left(r_{j}-|w-1|\right)^{1 / k-1}|\mathrm{~d} w| \\
& <\int_{J} k^{-1} s_{n}^{-N_{3}}\left|f(u)-a_{j}\right|^{-1 / k}\left(r_{j}-|w-1|\right)^{1 / k-1}|\mathrm{~d} w| \\
& =\int_{|f(u)-1|}^{r_{j}} k^{-1} s_{n}^{-N_{3}}\left|f(u)-a_{j}\right|^{-1 / k}\left(r_{j}-t\right)^{1 / k-1} \mathrm{~d} t \\
& =s_{n}^{-N_{3}}\left|f(u)-a_{j}\right|^{-1 / k}\left(r_{j}-|f(u)-1|\right)^{1 / k}=s_{n}^{-N_{3}} .
\end{aligned}
$$

Lemma 5.7 Let $E_{p}$ be a component of $E \cap K_{n}$ which meets $L_{n}$, and suppose that there exists $j=j(p)$ such that $E_{p}$ contains $k$ points $\zeta_{1}, \ldots, \zeta_{k} \in D_{j}$, each with $\left|f\left(\zeta_{q}\right)-a_{j}\right| \leq s_{n}^{-N_{7}}$. Assume further that $\left|\zeta_{q}-\zeta_{q^{\prime}}\right| \geq s_{n}^{-N_{3}}$ for $q \neq q^{\prime}$. Then $\left|f(z)-a_{j}\right| \leq s_{n}^{-N_{2}}$ for all $z \in E_{p}$, and $E_{p} \subseteq C(\varepsilon)$.

Proof Let $M_{0}=\sup \left\{|f(z)|: z \in E_{p}\right\}$; then $M_{0}<+\infty$ since poles of $f$ in $\mathbb{C} \backslash\{0\}$ are poles of $G$, by (5.3), and $|G(z)| \leq \eta$ on the closure of $E_{p}$. Choose $u_{0} \in E_{p}$ with $\left|f\left(u_{0}\right)\right| \geq M_{0} / 2$. There exists a polynomial $P$, of degree at most $k-1$, such that

$$
f(z)=P(z)+\int_{u_{0}}^{z} \frac{(z-t)^{k-1}}{(k-1) !} f^{(k)}(t) \mathrm{d} t \quad \text { on } E_{p} .
$$

The length of the boundary of $E_{p}$ is at most $2 s_{n}^{N_{1}}$ by (5.4). Hence each $z \in E_{p}$ can be joined to $u_{0}$ by a path in the closure of $E_{p}$, of length at most $4 s_{n}^{N_{1}}$, and so

$$
|f(z)-P(z)| \leq M_{0} \eta t_{n}^{-N_{5}}\left(2 T_{n}\right)^{k-1} 4 s_{n}^{N_{1}} \leq M_{0} s_{n}^{-N_{4}},
$$

by (5.3) and (5.7). In particular, this gives $\left|P\left(\zeta_{q}\right)-a_{j}\right| \leq\left(1+M_{0}\right) s_{n}^{-N_{4}}$ for each $q$. For $z$ in $E_{p}$, Lagrange's interpolation formula leads to

$$
\begin{aligned}
\left|P(z)-a_{j}\right| & =\left|\sum_{q=1}^{k}\left(P\left(\zeta_{q}\right)-a_{j}\right) \prod_{\nu \neq q} \frac{z-\zeta_{\nu}}{\zeta_{q}-\zeta_{\nu}}\right| \\
& \leq k\left(1+M_{0}\right) s_{n}^{-N_{4}}\left(2 T_{n}\right)^{k-1} s_{n}^{(k-1) N_{3}} \leq\left(1+M_{0}\right) s_{n}^{-N_{3}} .
\end{aligned}
$$

Setting $z=u_{0}$ in (5.11) then delivers $M_{0} \leq 2\left|P\left(u_{0}\right)\right| \leq 2\left|a_{j}\right|+o\left(1+M_{0}\right)$ and so $M_{0} \leq 5$. Now combining (5.10) with (5.11) yields $\left|f(z)-a_{j}\right| \leq s_{n}^{-N_{2}}$ and hence $|f(z)-1|<\varepsilon$ on $E_{p}$. Since $E_{p}$ meets $D_{j} \subseteq C(\varepsilon)$, this gives $E_{p} \subseteq C(\varepsilon)$. 
For each $j \in\left\{1, \ldots, N_{8}\right\}$ choose $\lambda=s_{n}^{N_{2}}$ points $u_{j, 1}, \ldots, u_{j, \lambda}$ on $\sigma_{j}$, each with $s_{n} / 2 \leq\left|u_{j, \kappa}\right| \leq s_{n}$ and such that $\left|u_{j, \kappa+1}\right| \geq\left|u_{j, \kappa}\right|+2 s_{n}^{-N_{3}}$. Applying Lemma 5.6 with $u=u_{j, \kappa}$ gives points $v_{j, \kappa} \in \sigma_{j}$ with $s_{n} / 2 \leq\left|u_{j, \kappa}\right| \leq\left|v_{j, \kappa}\right| \leq\left|u_{j, \kappa}\right|+s_{n}^{-N_{3}} \leq 2 s_{n}$ and, using (5.3), (5.5) and (5.9),

$$
\left|f\left(v_{j, \kappa}\right)-a_{j}\right| \leq s_{n}^{-N_{7}}, \quad\left|G\left(v_{j, \kappa}\right)\right| \leq 2\left|v_{j, \kappa}\right|^{N_{5}}\left|f^{(k)}\left(v_{j, \kappa}\right)\right| \leq s_{n}^{-N_{6}}<\eta .
$$

These points $v_{j, \kappa}$ satisfy $\left|v_{j, \kappa+1}\right| \geq\left|v_{j, \kappa}\right|+s_{n}^{-N_{3}}$, and each lies in a component of $E \cap K_{n}$ which meets $L_{n}$. Since there are $s_{n}^{N_{2}}$ of these $v_{j, \kappa}$ for each $j$, but at most $s_{n}^{N_{1}}$ available components $E_{p}$ by Lemma 5.5, it must be the case that for each $j$ there are at least $k$ points $v_{j, \kappa}$ lying in the same component $E_{p}$. Lemma 5.7 then implies that $E_{p} \subseteq C(\varepsilon)$ and $f(z)=a_{j}+o(1)$ on $E_{p}$.

Thus for $j=1, \ldots, N_{8}$ the following exist: a component $C_{j}=E_{p_{j}} \subseteq C(\varepsilon)$ of $E \cap K_{n}$ which meets $L_{n}$ and on which $f(z)=a_{j}+o(1)$; a point $v_{j} \in C_{j}$ such that, by (5.12),

$$
s_{n} / 2 \leq\left|v_{j}\right| \leq 2 s_{n}, \quad\left|G\left(v_{j}\right)\right| \leq s_{n}^{-N_{6}} .
$$

Since $C_{j} \subseteq C(\varepsilon)$, the function $\log |1 / G(z)|$ is subharmonic on $C_{j}$. Moreover, because $j^{\prime} \neq j$ gives $f(z) \rightarrow a_{j^{\prime}} \neq a_{j}$ as $z \rightarrow \infty$ on $\sigma_{j^{\prime}}$, the $C_{j}$ are pairwise disjoint and none of them contains a circle $S(0, t)$ with $t \in\left[t_{n}, T_{n}\right]$. For $t>0$ let $\phi_{j}(t)$ be the angular measure of $C_{j} \cap S(0, t)$. Then (5.7) and [20, p. 116] give a harmonic measure estimate

$$
\begin{aligned}
\omega\left(v_{j}, C_{j}, S\left(0, T_{n}\right) \cup S\left(0, t_{n}\right)\right) \leq & c_{1} \exp \left(-\pi \int_{2\left|v_{j}\right|}^{T_{n} / 2} \frac{\mathrm{d} t}{t \phi_{j}(t)}\right) \\
& +c_{1} \exp \left(-\pi \int_{2 t_{n}}^{\left|v_{j}\right| / 2} \frac{\mathrm{d} t}{t \phi_{j}(t)}\right),
\end{aligned}
$$

for $j=1, \ldots, N_{8}$, in which $c_{1}$ is a positive absolute constant. By Lemma 3.3 and (5.7), there exists at least one $j$ for which $\omega\left(v_{j}, C_{j}, S\left(0, T_{n}\right) \cup S\left(0, t_{n}\right)\right) \leq 2 c_{1} s_{n}^{-N_{7}}$. For this choice of $j$ the two constants theorem [18] delivers, using (5.8), (5.13) and the fact that $|G(z)|=\eta$ on $\partial C_{j} \cap K_{n}$,

$$
N_{6} \log s_{n} \leq \log \frac{1}{\left|G\left(v_{j}\right)\right|} \leq \log \frac{1}{\eta}+2 c_{1} s_{n}^{-N_{7}+N_{1}+1},
$$

a contradiction since $n$ is large.

\section{Proof of Theorem 1.4}

This is almost identical to the corresponding proof in [2], but with Theorem 1.3 standing in for the Denjoy-Carleman-Ahlfors theorem. Suppose that $f, k$ and $\alpha$ are 
as in the hypotheses, but there exists $\varepsilon>0$ such that in the neighbourhood $C(\varepsilon)$ of the singularity the function $f^{\prime} f^{(k)}$ has finitely many zeros which are not $\alpha$-points of $f$ : it may be assumed that there are no such zeros. On the other hand, because the singularity is indirect, $f$ must have infinitely many $\alpha$-points in $C(\varepsilon)$. Since $f^{(k)} / f$ has finite lower order, $f^{-1}$ cannot have infinitely many direct transcendental singularities over finite non-zero values, by Theorem 1.3. Set $A(\varepsilon)=\{w \in \mathbb{C}: 0<|w-\alpha|<\varepsilon\}$ if $\alpha \in \mathbb{C}$, with $A(\varepsilon)=\{w \in \mathbb{C}:|w|>1 / \varepsilon\}$ if $\alpha=\infty$. In either case, it may be assumed that $\varepsilon$ is so small that $A(\varepsilon) \subseteq \mathbb{C} \backslash\{0\}$ and there is no $w$ in $A(\varepsilon)$ such that $f^{-1}$ has a direct transcendental singularity over $w$.

Take $z_{0} \in C(\varepsilon)$, with $f\left(z_{0}\right)=w_{0} \neq \alpha$, and let $g$ be that branch of $f^{-1}$ mapping $w_{0}$ to $z_{0}$. If $g$ admits unrestricted analytic continuation in $A(\varepsilon)$ then, exactly as in [2], the classification theorem from [18, p. 287] shows that $z_{0}$ lies in a component $C_{0}$ of the set $\{z \in \mathbb{C}: f(z) \in A(\varepsilon) \cup\{\alpha\}\}$ which contains at most one point $z$ with $f(z)=\alpha$, so that $C(\varepsilon) \nsubseteq C_{0}$. But any $z_{1} \in C(\varepsilon)$ can be joined to $z_{0}$ by a path $\lambda$ on which $f(z) \in A(\varepsilon) \cup\{\alpha\}$, which gives $\lambda \subseteq C_{0}$ and hence $C(\varepsilon) \subseteq C_{0}$, a contradiction.

Hence there exists a path $\gamma:[0,1] \rightarrow A(\varepsilon)$, starting at $w_{0}$, such that analytic continuation of $g$ along $\gamma$ is not possible. This gives rise to $S \in[0,1]$ such that, as $t \rightarrow S-$, the image $z=g(\gamma(t))$ either tends to infinity or to a zero $z_{2} \in C(\varepsilon)$ of $f^{\prime}$ with $f\left(z_{2}\right)=\gamma(S) \in A(\varepsilon)$, the latter impossible by assumption. It follows that setting $z=\sigma(t)=g(\gamma(t))$, for $0 \leq t<S$, defines a path $\sigma$ tending to infinity in $C(\varepsilon)$, on which $f(z) \rightarrow w_{1} \in A(\varepsilon)$ as $z \rightarrow \infty$. But then there exists $\delta>0$ such that an unbounded subpath of $\sigma$ lies in a component $C^{\prime} \subseteq C(\varepsilon)$ of the set $\left\{z \in \mathbb{C}:\left|f(z)-w_{1}\right|<\delta\right\}$, with $\delta$ so small that $f^{\prime} f^{(k)}$ has no zeros on $C^{\prime}$. Further, the singularity over $w_{1}$ must be indirect, since direct singularities over values in $A(\varepsilon)$ have been excluded, and this contradicts Proposition 5.1.

\section{A Result Needed for Theorem 1.6}

Theorem 7.1 [16, Theorem 1] Let $u$ be a subharmonic function in the plane such that $B(r)=\sup \{u(z):|z|=r\}$ satisfies $\lim _{r \rightarrow \infty}(\log r)^{-1} B(r)=+\infty$. Then there exist $\delta_{0}>0$ and a simple path $\gamma:[0, \infty) \rightarrow \mathbb{C}$ with $\gamma(t) \rightarrow \infty$ as $t \rightarrow+\infty$ and the following properties:

(i) $\lim _{z \rightarrow \infty, z \in \gamma} \frac{u(z)}{\log |z|}=+\infty$; (ii) if $\lambda>0$ then $\int_{\gamma} \exp (-\lambda u(z))|\mathrm{d} z|<\infty$;

(iii) if $z=\gamma(t)$ then $u(\gamma(s)) \geq \delta_{0} u(z)$ for all $s \geq t$.

Conclusion (iii) and the fact that $\gamma$ may be chosen to be simple are not stated in [16, Theorem 1], but both are implicit in the proof. Here $\gamma=\gamma_{1} \cup \gamma_{2} \cup \ldots$ is constructed in [16, Section 3] so that, for some fixed $\delta_{1} \in(0,1)$, each $\gamma_{k}:[k-1, k] \rightarrow \mathbb{C}$ is a simple path from $a_{k} \in D_{k}$ to $a_{k+1} \in \partial D_{k}$, where $D_{k}$ is the component of $\left\{z \in \mathbb{C}: u(z)<\left(1-\delta_{1}\right)^{-1} u\left(a_{k}\right)\right\}$ containing $a_{1}$. By [16, (3.2) and (3.3)], the $\gamma_{k}$ are such that $0<\delta_{1} u\left(a_{k}\right) \leq u(z)<\left(1-\delta_{1}\right)^{-1} u\left(a_{k}\right)$ on $\lambda_{k}=\gamma_{k} \backslash\left\{a_{k+1}\right\}$ and $u\left(a_{k+1}\right) \geq\left(1-\delta_{1}\right)^{-1} u\left(a_{k}\right)>u\left(a_{k}\right)$. Hence if $z=\gamma(t) \in \lambda_{k}$, then $u(\gamma(s)) \geq$ $\delta_{1} u\left(a_{k}\right) \geq \delta_{1}\left(1-\delta_{1}\right) u(\gamma(t))$ for all $s \geq t$. If the whole path $\gamma$ is not simple, take the 
least $k \geq 2$ such that $\Gamma_{k}=\gamma_{1} \cup \ldots \cup \gamma_{k}$ is not simple. Then there exists a maximal $t \in[k-1, k]$ such that $u_{k}=\gamma_{k}(t)$ lies in the compact set $\Gamma_{k-1}$, and $t<k$ since $\gamma_{k}(k)=a_{k+1} \in \partial D_{k}$. Replacing $\Gamma_{k}$ by the part of $\Gamma_{k-1}$ from $a_{1}$ to $u_{k}$, followed by the part of $\gamma_{k}$ from $u_{k}$ to $a_{k+1}$, does not affect conclusions (i), (ii) and (iii), and the argument may then be repeated.

Theorem 7.1 leads to the following result.

Proposition 7.1 Let $N \in \mathbb{N}$ and let $A$ be a transcendental meromorphic function in the plane such that the inverse function of A has a direct transcendental singularity over 0 . Then there exists a path $\gamma$ tending to infinity in $\mathbb{C}$ and linearly independent solutions $U, V$ of

$$
w^{\prime \prime}+A(z) w=0
$$

on a simply connected domain containing $\gamma$, such that $U$ and $V$ satisfy, as $z \rightarrow \infty$ on $\gamma$,

$$
U(z)=z+\frac{O(1)}{z^{N}}, \quad U^{\prime}(z)=1+\frac{O(1)}{z^{N}}, \quad V(z)=1+\frac{O(1)}{z^{N}}, \quad V^{\prime}(z)=\frac{O(1)}{z^{N}} .
$$

To prove Proposition 7.1, observe first that, as in the proof of Theorem 1.3, there exist a small positive $\delta$ and a non-empty component $D$ of $\{z \in \mathbb{C}:|A(z)|<\delta\}$ such that $A(z) \neq 0$ on $D$, as well as a non-constant subharmonic function $u$ on $\mathbb{C}$ given by

$$
u(z)=\log \left|\frac{\delta}{A(z)}\right| \quad(z \in D), \quad u(z)=0 \quad(z \notin D) .
$$

Then $u$ satisfies the hypotheses of Theorem 7.1, by [4, Theorem 2.1], and so there exists a path $\gamma:[0, \infty) \rightarrow D$ as in conclusions (i), (ii) and (iii). In particular, (iii) implies that

$$
\text { if } z=\gamma(t) \text { then }|A(\gamma(s))| \leq \delta^{1-\delta_{0}}|A(z)|^{\delta_{0}} \text { for all } s \geq t \text {. }
$$

Choose a simply connected domain $\Omega$ on which $A$ has no poles, such that $\gamma \subseteq \Omega$. By (7.1) it may be assumed that $|A(t)|^{-1 / 4} \geq|t|^{2} \geq 4$ on $\gamma$, and that

$$
\int_{\gamma}|t|^{2}|A(t)||\mathrm{d} t| \leq \int_{\gamma}|t|^{2}|A(t)|^{1 / 2}|\mathrm{~d} t| \leq \int_{\gamma}|A(t)|^{1 / 4}|\mathrm{~d} t|<\frac{1}{4} .
$$

Lemma 7.1 Let $v$ be a solution of (7.2) on $\Omega$. Then $v(z)=O(|z|)$ as $z \rightarrow \infty$ on $\gamma$.

Proof This is a standard argument along the lines of Gronwall's lemma. Let $y_{0}$ be the starting point of $\gamma$. Differentiating twice shows that there exist constants $a_{1}, b_{1}$ such that, on $\Omega$,

$$
v(z)=a_{1} z+b_{1}-\int_{y_{0}}^{z}(z-t) A(t) v(t) \mathrm{d} t
$$


If $\phi(z)=v(z) / z$ is unbounded on $\gamma$, there exist $\zeta_{n} \rightarrow \infty$ on $\gamma$ such that $\phi\left(\zeta_{n}\right) \rightarrow \infty$ and $|\phi(t)| \leq\left|\phi\left(\zeta_{n}\right)\right|$ on the part of $\gamma$ joining $y_{0}$ to $\zeta_{n}$. If $n$ is large then (7.5) delivers a contradiction via

$$
\left|\phi\left(\zeta_{n}\right)\right| \leq\left|a_{1}\right|+\left|b_{1}\right|+\left|\phi\left(\zeta_{n}\right)\right| \int_{y_{0}}^{z}(1+|t|)|t A(t)||\mathrm{d} t| \leq\left|a_{1}\right|+\left|b_{1}\right|+\frac{\left|\phi\left(\zeta_{n}\right)\right|}{2} .
$$

Lemma 7.2 (a) Let $N \in \mathbb{N}$. Then on $\gamma$ every solution $v_{j}$ of (7.2) has

$$
v_{j}(z)=\alpha_{j} z+\beta_{j}+\int_{z}^{\infty}(z-t) A(t) v_{j}(t) \mathrm{d} t, \quad \alpha_{j}, \beta_{j} \in \mathbb{C},
$$

the integration being from $z$ to infinity along $\gamma$. Moreover, $v_{j}$ satisfies, as $z \rightarrow \infty$ on $\gamma$,

$$
v_{j}(z)-\alpha_{j} z-\beta_{j}=\frac{O(1)}{z^{N}}, \quad v_{j}^{\prime}(z)-\alpha_{j}=\frac{O(1)}{z^{N}} .
$$

(b) If $v_{1}, v_{2}$ are linearly independent solutions of (7.2) on $\Omega$ then $\left|\alpha_{1}\right|+\left|\alpha_{2}\right|>0$ in (7.6), and if $\alpha_{2}=0$ then $\beta_{2} \neq 0$.

Proof First, (7.6) follows from (7.5) and Lemma 7.1. Next, (7.1), (7.4)-(7.6) and Lemma 7.1 imply that, as $z \rightarrow \infty$ on $\gamma$,

$$
\begin{aligned}
\left|v_{j}(z)-\alpha_{j} z-\beta_{j}\right| & \leq|z| \int_{z}^{\infty}(1+|t|)|A(t)| O(|t|)|\mathrm{d} t| \\
& \leq|z| \delta^{\left(1-\delta_{0}\right) / 2}|A(z)|^{\delta_{0} / 2} \int_{z}^{\infty}(1+|t|)|A(t)|^{1 / 2} O(|t|)|\mathrm{d} t|=\frac{O(1)}{z^{N}} \\
\left|v_{j}^{\prime}(z)-\alpha_{j}\right| & =\left|\int_{z}^{\infty} A(t) v_{j}(t) \mathrm{d} t\right| \\
& \leq \delta^{\left(1-\delta_{0}\right) / 2}|A(z)|^{\delta_{0} / 2} \int_{z}^{\infty}|A(t)|^{1 / 2} O(|t|)|\mathrm{d} t|=\frac{O(1)}{z^{N}}
\end{aligned}
$$

Finally, suppose that $v_{1}, v_{2}$ are linearly independent solutions of (7.2) on $\Omega$, but the conclusion of (b) fails. Then $v_{1}(z) v_{2}^{\prime}(z)-v_{1}^{\prime}(z) v_{2}(z) \rightarrow 0$ as $z \rightarrow \infty$ on $\gamma$, by (7.7), contradicting the fact that $W\left(v_{1}, v_{2}\right)$ is a non-zero constant by Abel's identity.

Now fix linearly independent solutions $v_{1}, v_{2}$ of (7.2) on $\Omega$. Then $\alpha_{1}, \alpha_{2}$ cannot both vanish in (7.6). On the other hand, it is possible to ensure that one of $\alpha_{1}, \alpha_{2}$ is 0 , by otherwise considering $\alpha_{2} v_{1}-\alpha_{1} v_{2}$. Hence it may be assumed that $\alpha_{1}=1$, while $\alpha_{2}=0$ and $\beta_{2}=1$. Now write $U=v_{1}$ and $V=v_{2}$, so that Lemma 7.2 gives (7.3). 


\section{Proof of Theorem 1.6}

Assume that $f$ and $S_{f}$ are as in the hypotheses, but that the inverse function of $S_{f}$ has a direct transcendental singularity over 0 . Then evidently so has that of $A=S_{f} / 2$, and it is well known that (1.3) implies that $f$ is locally the quotient of linearly independent solutions of (7.2). Now Proposition 7.1 gives linearly independent solutions $U, V$ of (7.2) satisfying (7.3) on a path $\gamma$ tending to infinity. Moreover, $h=U / V$ has the form $h=T \circ f$, for some Möbius transformation $T$, and so $h \in \mathcal{S}$, whereas $h(z) \sim z$ and $z h^{\prime}(z) / h(z)=O(1)$ on $\gamma$, contradicting (3.5).

Open Access This article is distributed under the terms of the Creative Commons Attribution 4.0 International License (http://creativecommons.org/licenses/by/4.0/), which permits unrestricted use, distribution, and reproduction in any medium, provided you give appropriate credit to the original author(s) and the source, provide a link to the Creative Commons license, and indicate if changes were made.

\section{References}

1. Bergweiler, W.: Iteration of meromorphic functions. Bull. Am. Math. Soc. 29, 151-188 (1993)

2. Bergweiler, W., Eremenko, A.: On the singularities of the inverse to a meromorphic function of finite order. Rev. Mat. Iberoam. 11, 355-373 (1995)

3. Bergweiler, W., Eremenko, A.: Direct singularities and completely invariant domains of entire functions. Ill. J. Math. 52, 243-259 (2008)

4. Bergweiler, W., Rippon, P.J., Stallard, G.M.: Dynamics of meromorphic functions with direct or logarithmic singularities. Proc. Lond. Math. Soc. 97, 368-400 (2008)

5. Clunie, J., Eremenko, A., Rossi, J.: On equilibrium points of logarithmic and Newtonian potentials. J. Lond. Math. Soc 47(2), 309-320 (1993)

6. Elfving, G.: Über eine Klasse von Riemannschen Flächen und ihre Uniformisierung. Acta Soc. Sci. Fenn. 2, 1-60 (1934)

7. Hayman, W.K.: Picard values of meromorphic functions and their derivatives. Ann. Math. 70, 9-42 (1959)

8. Hayman, W.K.: Meromorphic Functions. Clarendon Press, Oxford (1964)

9. Hayman, W.K.: Subharmonic Functions, vol. 2. Academic Press, London (1989)

10. Hayman, W.K.: Multivalent Functions. Cambridge Tracts in Mathematics, vol. 110, 2nd edn. Cambridge University Press, Cambridge (1994)

11. Hinchliffe, J.D.: The Bergweiler-Eremenko theorem for finite lower order. Results Math. 43, 121-128 (2003)

12. Langley, J.K.: The zeros of the first two derivatives of a meromorphic function. Proc. Am. Math. Soc. 124(8), 2439-2441 (1996)

13. Langley, J.K.: Non-real zeros of higher derivatives of real entire functions of infinite order. J. d'Analyse Math. 97, 357-396 (2005)

14. Langley, J.K.: The Schwarzian derivative and the Wiman-Valiron property. J. d'Analyse Math. 130, 71-89 (2016)

15. Langley, J.K., Rossi, John: Critical points of certain discrete potentials. Complex Var. 49, 621-637 (2004)

16. Lewis, J., Rossi, J., Weitsman, A.: On the growth of subharmonic functions along paths. Ark. Mat. 22, 104-114 (1983)

17. Nevanlinna, R.: Über Riemannsche Flächen mit endlich vielen Windungspunkten. Acta Math. 58, 295-373 (1932)

18. Nevanlinna, R.: Eindeutige analytische Funktionen, 2nd edn. Springer, Berlin (1953)

19. Sixsmith, D.J.: A new characterisation of the Eremenko-Lyubich class. J. d'Analyse Math. 123, 95-105 (2014)

20. Tsuji, M.: Potential Theory in Modern Function Theory. Maruzen, Tokyo (1959) 